\title{
Anticancer bioactive peptide combined with docetaxel and its mechanism in the treatment of breast cancer
}

\author{
XIAN LI* BEIBEI GAO* and XIULAN SU \\ Clinical Medicine Research Center, The Affiliated Hospital of Inner Mongolia Medical University, \\ Hohhot, Inner Mongolia 010050, P.R. China
}

Received May 22, 2019; Accepted February 7, 2020

DOI: $10.3892 / \mathrm{etm} .2020 .8902$

\begin{abstract}
Breast cancer remains a worldwide public-health issue. Novel drugs that increase the sensitivity and reduce the toxic side effects of chemotherapeutic agents are urgently required. The present study investigated the effect and mechanism of the short-term intermittent administration of an anticancer bioactive peptide (ACBP), docetaxel (DTX), ACBP combined with DTX (MIX) and ACBP combined with low dose DTX (L-MIX) to nude mice bearing human breast cancer tumors. The body weight, tumor length, tumor diameter, diet and water consumption of the tumor-bearing nude mice were calculated. The protein and mRNA expression levels of p53, p21 and Ki67 were detected via immunohistochemistry and reverse transcription-quantitative PCR, respectively. The results revealed that the activity level of each group of mice was consistent. However, the food and water consumption of the ACBP group was significantly increased compared with the NS group. Compared with the normal saline group, the tumor weights and volumes of the treatment groups were significantly decreased, indicating an inhibitory effect of the treatment. However, the MIX group exhibited lower tumor weights and volumes compared with the ACBP and DTX groups. Furthermore, no significant cell necrosis, edema or inflammatory cell infiltration was observed upon hematoxylin $\&$ eosin staining of the liver and spleen in all groups. The results also revealed that the p21, p53 and Ki67 protein and mRNA levels were decreased in the ACBP, DTX and MIX groups compared with the control group. Additionally, when compared with those in the MIX and L-MIX groups, the p21 and Ki67 protein, and p53 and Ki67 mRNA levels in the
\end{abstract}

Correspondence to: Professor Xiulan Su, Clinical Medicine Research Center, The Affiliated Hospital of Inner Mongolia Medical University, 1 Tongdao North Street, Hohhot, Inner Mongolia 010050, P.R. China

E-mail:xlsu@hotmail.com

${ }^{*}$ Contributed equally

Key words: anticancer bioactive peptide, docetaxel, chemotherapy drugs, p53-p21 signaling pathway, breast cancer
ACBP and DTX groups were significantly increased. The results suggested that the short-term intermittent use of ACBP alone had an inhibitory effect on tumor growth and improved the food and water consumption of tumor-bearing nude mice. Furthermore, the combination of ACBP and DTX reduced toxic side effects and the dosage requirement of drugs to achieve therapeutic effects on the tumor-bearing nude mice. Therefore, the antitumor effect of ACBP may be associated with the improvement of immune function in tumor-bearing nude mice and ACBP may serve an antitumor role via the p53-p21 signaling pathway in breast cancer.

\section{Introduction}

The incidence of breast cancer is increasing and shifting to younger populations worldwide, becoming the main cause of cancer-associated death in females (1). In 2012, there were an estimated 1.66 million new breast cancer cases (25\% of all cancer cases) and 521,000 breast cancer-associated deaths (2). In 2015 , breast cancer accounted for $\sim 15 \%$ of newly diagnosed cancer cases in China (3). In Western countries, breast cancer primarily occurs in 55-60-year-old women, but the trend of onset in China is significantly earlier, occurring primarily in 45-55-year-old middle-aged and young women (4). The occurrence of breast cancer in younger women has threatened the work, quality of life and health of those affected (5). Currently, the main treatment strategies for breast cancer are surgery, radiotherapy, chemotherapy, endocrine therapy and molecular-targeted therapy, which are all auxiliary (6). However, the side effects of chemotherapy in patients remain serious, with $39.7 \%$ of patients suffering from neutropenia and infection (7). The cardiotoxicity of chemotherapeutic drugs is a well-known major adverse reaction affecting the quality of life and mortality of patients with cancer (8). Anticancer drugs have been reported to cause various types of cardiac toxicity, including heart failure, bradycardia, prolonged QT intervals, myocardial ischemia and cardiomyopathy (9). Neurotoxicity is characterized by numbness and tingling of the fingers and toes, as well as myalgia and myasthenia (10). Due to the aforementioned toxic and adverse effects of chemotherapeutic drugs, a number of patients have to reduce their dosage and prolong the chemotherapy cycle (11). Furthermore, chemotherapy cessation leads to treatment failure (11). While prolonged survival remains the primary goal of chemotherapy, symptom relief and 
quality of life are also important treatment considerations (12). Therefore, bioactive peptides are rapidly being developed as potential anticancer drugs, which may reduce the side effects and increase the sensitivity of traditional chemotherapeutic drugs without altering their anticancer effect (13).

A wide range of bioactive peptides exist in natural resources, including specific small molecular protein fragments that, although inactive within the protein sequence, can be released during proteolysis or fermentation and are activated by the digestive, endocrine, cardiovascular, immune and nervous systems, which serve as important roles in human health (14). Several peptides released from animal proteins have been reported to have different health effects in vitro and in vivo, including antimicrobial properties, blood pressure reduction, cholesterol reduction, antithrombotic and antioxidant activity and opioid-like activity (14-16). These peptides have also been reported to enhance mineral absorption and bioavailability, exhibit cellular and immunomodulatory effects and exhibit antiobesity and antigenotoxic activities (14-16). Bioactive peptides have low immunogenicity, excellent tissue penetration, low production costs and are easy to modify to enhance their stability and biological activity within the body, making these molecules ideal candidates for cancer therapy (17). The anticancer bioactive peptide (ACBP) used in the present study is a low molecular weight bioactive substance extracted from goat spleen following induced immunization (relative molecular weight, $<8000$ Da; patent no. ZL961222236.0), which is a novel method of anticancer biological preparation. A previous study has reported that ACBP inhibits tumor angiogenesis, regulates protein degradation, interferes with DNA synthesis, regulates the cell cycle, induces apoptosis and influences further antitumor mechanisms (18). A large number of previous celland animal-based experiments reported that ACBP served an inhibitory effect on the BGC-823 and MGC-803 human gastric cancer cell lines, the MKN-45 leukemia cell line, the $\mathrm{H}-22$ hepatoma cell line, the CNE nasopharyngeal carcinoma cell line and the GBC-SD gallbladder cancer cell line (19-21). Collectively, these aforementioned studies suggested that ACBP may be a potential tumor stem cell-targeted drug and when combined with chemotherapeutic drugs, ACBP may effectively improve their therapeutic efficacy and reduce their toxicity in patients (22).

Docetaxel (DTX) is an effective anticancer agent that is widely used and has demonstrated extensive anticancer activity against breast, lung, pancreatic, prostate, ovarian and head and neck cancer (23-26). DTX is one of the most commonly used chemotherapy drugs for breast cancer (23). DTX binds to the $\beta$-subunit of microtubule proteins, leading to stable and non-functional microtubule formation by promoting polymerization and inhibiting decomposition, ultimately resulting in mitosis arrest and apoptosis induction (10). Therefore, the present study investigated the effect of the intermittent short-term application of ACBP and ACBP combined with DTX, on the quality of life of nude mice bearing human breast cancer tumors. Furthermore, the expression of p53, p21 and Ki67 were assessed. The effect of ACBP on the human breast cancer cell line MDA-MB-231 in nude mice, as well as the toxicity-reducing and sensitivity-increasing mechanisms of ACBP were also studied.

\section{Materials and methods}

Cell lines and mice. All animal experiments were approved by the Ethics Committee for Animal Experiments of Inner Mongolia Medical College (approval no. YKD2016152). A total of 40 female Balb/c-Nu nude mice (age, 4-6 weeks; weight, $16 \pm 2 \mathrm{~g}$ ) of specific pathogen-free grade were used. The animals were purchased from Beijing Weitong Lihua Experimental Animal Technology Co., Ltd. [license no. SCXK (Beijing) 2012-0001]. The human breast cancer cell line MDA-MB-231 was purchased from the China Infrastructure of Cell Line Resources, Institute of Basic Medical Sciences, Chinese Academy of Medical Sciences.

Establishment of an axillary-transplanted tumor model using the human breast cancer cell line MDA-MB-231 in nude mice. The human breast cancer cell line, MDA-MB-231, was cultured in DMEM (Thermo Fisher Scientific, Inc.) supplemented with $10 \%$ fetal calf serum (HyClone; GE Healthcare Life Sciences) and $1 \%$ penicillin-streptomycin solution (Thermo Fisher Scientific, Inc.) in a humidified atmosphere of $5 \% \mathrm{CO}_{2}$ at $37^{\circ} \mathrm{C}$. The cells in the logarithmic growth phase were selected for further experiments. A single-cell suspension of $5 \times 10^{7} / \mathrm{ml}$ was used for injection.

Mice were maintained at $20-25^{\circ} \mathrm{C}$ with $40-70 \%$ humidity and free access to food and drinking water. Animals were also housed under a $12 \mathrm{~h}$ light/dark cycle. Eating, feeding and operating procedures strictly followed aseptic principles. After 3 days of free access to food in nude mice, a $0.1 \mathrm{ml}$ single-cell suspension was inoculated into the right axilla of the 40 nude mice. The activity and tumorigenesis of the nude mice were observed daily. At 6 days post-injection, rice-sized nodules were identified at the injection site of the nude mice and the tumor formation rate was $100 \%$. Therefore, the breast cancer model was successfully established in nude mice.

Experimental grouping, administration and observation records. The 40 nude mice were randomly divided into five groups ( $\mathrm{n}=8)$ as follows: Normal saline group (NS), anticancer bioactive peptide group (ACBP), docetaxel group (DTX), ACBP combined DTX treatment group (MIX) and ACBP combined with low dose DTX treatment group (L-MIX). The treatments were administered by tail vein injection twice a week for a total of 3 weeks. The NS group was administered $0.4 \mathrm{ml}$ saline, the ACBP group was administered $0.4 \mathrm{ml}$ ACBP $(70 \mathrm{mg} / \mathrm{ml}$; Prepared by The Clinical Medical Research Center, Affiliated Hospital of Inner Mongolia Medical University), the DTX group was administered $5 \mathrm{mg} / \mathrm{kg}$ DTX $(0.5 \mathrm{ml} / 20 \mathrm{mg}$; Prepared by Jiangsu Hengrui Pharmaceutical Co., Ltd.; Chinese medicine standard no. H20020543), the MIX group was administere $0.4 \mathrm{ml}$ ACBP combined with $5 \mathrm{mg} / \mathrm{kg}$ DTX, and the L-MIX was administered $0.4 \mathrm{ml}$ ACBP combined with $2.5 \mathrm{mg} / \mathrm{kg}$ DTX. The activity status of the nude mice was observed daily. The weight and tumor boundaries were measured every other day to calculate the tumor volume (V) using the following formula: $\mathrm{V}=\mathrm{ab}^{2} / 2$, where $\mathrm{a}$ is tumor length and $b$ is the shortest tumor diameter. Food and water intake were measured every 2 days to calculate the quantity consumed. After 3 weeks, the retro-orbital blood of nude mice were collected following anesthesia and the nude mice were 
Table I. Primer sequences used for reverse transcription-quantitative PCR.

Sequence $\left(5^{\prime} \rightarrow 3^{\prime}\right)$

\begin{tabular}{lll}
\cline { 2 - 3 } Gene & \multicolumn{1}{c}{ Forward } & \multicolumn{1}{c}{ Reverse } \\
\hline p53 & TCAACAAGATGTTTTGCCAACTG & ATGTGCTGTGACTGCTTGTAGATG \\
p21 & AAACTTTGGAGTCCCCTCAC & AAAGGCTCAACACTGAGACG \\
Ki67 & CTTGCCTCCTAATACGCCTCTC & CCTGACTCTTGTTTTCCTGATGGT \\
GAPDH & TCCACCACCCTGTTGCTGTA & ACCACAGTCCATGCCATCAC
\end{tabular}

subsequently euthanized by cervical dislocation. The tumor tissues, livers and spleens of the nude mice were isolated and weighed. The isolated tissues were divided into two parts: One was fixed in $4 \%$ paraformaldehyde for $24 \mathrm{~h}$ at $25^{\circ} \mathrm{C}$, and the other was stored at $-80^{\circ} \mathrm{C}$ until further analysis.

Determination of the liver and spleen coefficients. Following sacrifice, the body weights of the nude mice were measured. The livers and spleens were removed from the mice, dried with filter paper and were then weighed. The liver and spleen coefficients were calculated as follows: Liver coefficient=liver mass/nude mouse body mass and spleen coefficient $=$ spleen weight/body mass of nude mice.

Paraffin-embedded tissue sections and hematoxylin \& eosin staining. Tissues fixed in $4 \%$ paraformaldehyde were trimmed to a size of $5 \times 5 \times 3 \mathrm{~mm}^{3}$, placed in an embedding box, labeled and slowly flushed with flowing water overnight at $25^{\circ} \mathrm{C}$. The tumor tissue was dehydrated with an ascending ethanol series in 30 min increments as follows: $50 \%$ ethanol, 60\% ethanol, $70 \%$ ethanol, $80 \%$ ethanol, $95 \%$ ethanol I, $95 \%$ ethanol II, $95 \%$ ethanol I and anhydrous ethanol II at $25^{\circ} \mathrm{C}$. The liver tissue was dehydrated with an ascending ethanol series as follows: $50 \%$ ethanol for $15 \mathrm{~min}, 60 \%$ ethanol for $15 \mathrm{~min}, 70 \%$ ethanol for $15 \mathrm{~min}, 80 \%$ ethanol for $15 \mathrm{~min}, 95 \%$ ethanol I for $30 \mathrm{~min}$, $95 \%$ ethanol II for $30 \mathrm{~min}, 95 \%$ ethanol I for $30 \mathrm{~min}$ and anhydrous ethanol II for $30 \mathrm{~min}$ at $25^{\circ} \mathrm{C}$. Furthermore, the spleen tissue was dehydrated with an ascending ethanol series as follows: $50 \%$ ethanol for $10 \mathrm{~min}, 60 \%$ ethanol for $10 \mathrm{~min}$, $70 \%$ ethanol for $10 \mathrm{~min}, 80 \%$ ethanol for $10 \mathrm{~min}, 95 \%$ ethanol I for $30 \mathrm{~min}, 95 \%$ ethanol I for $30 \mathrm{~min}$, anhydrous ethanol II for $30 \mathrm{~min}$ and anhydrous ethanol II again for $30 \mathrm{~min}$ at $25^{\circ} \mathrm{C}$. The tissue sections were deparaffinized using xylene I for $20 \mathrm{~min}$ and xylene II for $20 \mathrm{~min}$ at $37^{\circ} \mathrm{C}$. Subsequently, the wax block was cut into $4-\mu \mathrm{m}$-thick slices for hematoxylin \& eosin staining for $2 \mathrm{~h}$ at $25^{\circ} \mathrm{C}$.

p53, p21 and Ki67 protein expression in transplanted tumor tissues as detected by immunohistochemistry. Streptavidin-peroxidase immunohistochemistry was performed to analyze the protein expression of p53, p21 and Ki67 in transplanted tumor tissues. For dewaxing (xylene I for $10 \mathrm{~min}$ and xylene II for $10 \mathrm{~min}$ ), hydration and antigen repair $\left(1,000 \mathrm{ml}\right.$ citrate buffer), the fragments were heated at $60^{\circ} \mathrm{C}$ for $1 \mathrm{~h}$ until the wax melted. Paraffin embedded sections were then rehydrated using a descending alcohol series. Subsequently, the endogenous peroxidase/phosphatase activity was blocked by incubating sections with $3 \% \mathrm{H}_{2} \mathrm{O}_{2}$ at room temperature for $9 \mathrm{~min}$. Sections were then treated with the following primary antibodies, washed three times for 3 mins with PBS and placed in a wet box overnight at $4^{\circ} \mathrm{C}$ : p53 (1:50; Abcam; cat. no. ab32049), p21 (1:50; Cell Signaling Technology, Inc.; cat. no. 2946) and Ki-67 (1:150; Abcam; cat. no. sc-9976). Subsequently, secondary antibodies (1:50; Abcam; cat. no. ab205718) were added to the slides for $30 \mathrm{~min}$ at room temperature. PBS was added to the sections for $3 \mathrm{~min}$ at $25^{\circ} \mathrm{C}$ to wash away the DAB (Abcam; cat. no. ab64238) chromogenic agent and subsequently, the slides were counterstained with hematoxylin $\&$ eosin for $5 \mathrm{~min}$ at $25^{\circ} \mathrm{C}$. The sections were slowly rinsed with running water for $5 \mathrm{sec}$, and $1 \%$ ethanol hydrochloride was mixed 10 times for cell differentiation. The samples were then rinsed with tap water for $30 \mathrm{sec}$. Subsequently, the samples were observed under a light microscope (magnification, x100) for statistical analysis by a semiquantitative scoring system. The staining intensity was scored as follows: No staining, 0; light yellow, 1; brown, 2; or tan, 3 . Four high power visual fields were randomly selected and the percentage of positive cells was determined as follows: No positive cells, 0 ; positive cells $<25 \%$, 1 ; positive cells $<25-49 \%, 2$; and positive cells $>50 \%, 3$. The final score was calculated as staining intensity $x$ percentage of positive cells. Scores $<3$ were defined as negative and scores $\geq 3$ were defined as positive (27).

Detection of p53, p21 and Ki67 mRNA expression in transplanted tumor tissues by reverse transcription-quantitative $P C R(R T-q P C R)$. Total RNA was extracted from the tumor tissues using TRIzol ${ }^{\circledR}$ reagent (Thermo Fisher Scientific, Inc.), according to the manufacturer's protocol. Pectrophotometric quantification was utilized to determine RNA purity using an OD ratio of $260 \mathrm{~nm} / 280 \mathrm{~nm}$ and a BeekmanDU $800 \mathrm{UV}$ spectrophotometer. A total of $1 \mathrm{mg}$ total RNA was reverse transcribed into first-strand cDNA using the Revert Aid First Strand cDNA Synthesis kit (Thermo Fisher Scientific, Inc.) according to the manufacturer's protocol. qPCR was performed using the Taq-Man ${ }^{\mathrm{TM}}$ Gene Expression assay (Applied Biosystems; Thermo Fisher Scientific, Inc.) on an Mx3000P real-time PCR system (Agilent Technologies, Inc.) according to the manufacturer's instructions. The primer pairs used in the present study were designed and synthesized by Sangon Biotech Co., Ltd. (Table I). The following thermocycling conditions were used: Initial denaturation at $95^{\circ} \mathrm{C}$ for $5 \mathrm{~min}$; 35 cycles of denaturation at $95^{\circ} \mathrm{C}$ for $10 \mathrm{sec}$, annealing at $60^{\circ} \mathrm{C}$ for $30 \mathrm{sec}$, elongation at $60^{\circ} \mathrm{C}$ for $30 \mathrm{sec}$ and a final extension step at $60^{\circ} \mathrm{C}$ for $40 \mathrm{sec}$. mRNA levels were normalized to the 
internal reference gene GAPDH. ${ }^{\Delta 4} \mathrm{CT}$ values of each group were calculated separately $=[(\mathrm{CT}$ experimental target gene CT internal reference target gene)-(CT control target gene-CT internal reference control gene)]. mRNA relative expression was calculated using $2^{-\Delta \Delta C q}$ values (28).

Statistical analysis. Statistical analyses were performed using SPSS software (version 19.0; IBM Corp.). Data are presented as the mean \pm standard deviation. Data containing two samples were analyzed using a Student's t-test. Comparisons in datasets containing $>3$ groups were evaluated by one-way ANOVA followed by Bonferroni post hoc test. $\mathrm{P}<0.05$ was considered to indicate a statistically significant difference.

\section{Results}

Food intake, water consumption and body weight of tumor-bearing nude mice. The levels of food intake and water consumption of thenude micein each groupexhibited significant differences. The ACBP group exhibited no significant dietary intake $(\mathrm{P}>0.05)$ and decreased drinking water consumption $(\mathrm{P}<0.01)$ compared with the NS group (Fig. 1A and B). In addition, the dietary intake and drinking water consumption were significantly reduced in the DTX and MIX groups compared with the NS group $(\mathrm{P}<0.05$; Fig. $1 \mathrm{~A}$ and $\mathrm{B})$. Furthermore, there was a statistically significant decrease in water consumption in the L-MIX group compared with the NS group $(\mathrm{P}<0.05)$. Each treatment group exhibited no significant difference in body weight compared with the NS group (Fig. 1C).

Tumor growth in nude mice. The treatment groups exhibited significantly decreased tumor weights and volumes compared with the NS group, indicating that the treatments had an inhibitory effect (Fig. 2A and B). The tumor weight of the NS, ACBP, DTX, MIX and L-MIX groups were $1.10 \pm 0.36$, $0.78 \pm 0.16,0.71 \pm 0.17,0.47 \pm 0.20$ and $0.66 \pm 0.24 \mathrm{~g}$, respectively (Fig. 2A). The tumor weights were significantly decreased in the DTX, MIX, L-MIX $(\mathrm{P}<0.05)$ and ACBP groups $(\mathrm{P}<0.01)$ compared with the NS group (Fig. 2A). Furthermore, the tumor volumes of the NS, ACBP, DTX, MIX and L-MIX groups were $1.82 \pm 0.70,0.77 \pm 0.24,0.65 \pm 0.29,0.42 \pm 0.24$ and $0.61 \pm 0.26 \mathrm{~mm}^{3}$, respectively (Fig. $2 \mathrm{~B}$ ). The tumor volumes in the ACBP and DTX groups were significantly reduced compared with the NS group $(\mathrm{P}<0.05$; Fig. $2 \mathrm{~B})$. Additionally, the tumor weights and volumes were lower in the MIX group, although not significantly different, compared with the ACBP and DTX groups, which suggested that ACBP not only exerted an inhibitory effect on the tumors alone, but also acted synergistically with DTX (Fig. 2A). The maximum tumor diameter observed was $\sim 10 \mathrm{~mm}$ and the maximum tumor volume observed was $\sim 2.5 \mathrm{~mm}^{3}$, both in the NS group (Fig. 2C). Therefore, the results suggested that ACBP inhibited tumor growth in breast cancer.

Liver and spleen tissue structure and coefficients in nude mice. The liver coefficients of the treatment groups exhibited no significant differences compared with the NS group (Fig. 3A). However, the ACBP group displayed a significantly higher spleen coefficient compared with the NS group ( $\mathrm{P}=0.041$; Fig. 3B). Furthermore, the spleen coefficients of the DTX, MIX
A

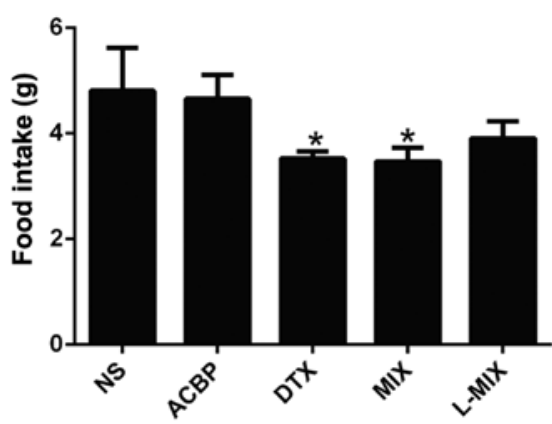

B

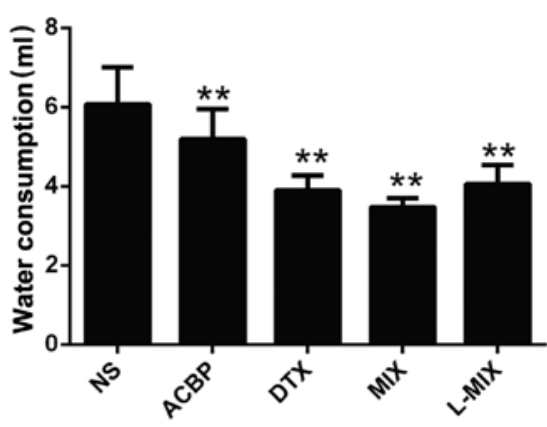

C

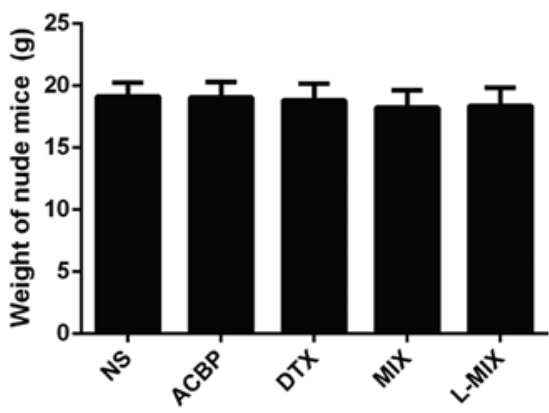

Figure 1. Behavioral characteristics and weight of tumor-bearing nude mice. The (A) food intake, (B) drinking water consumption and (C) weight of nude mice were calculated. ${ }^{*} \mathrm{P}<0.05$ and ${ }^{* *} \mathrm{P}<0.01$ vs. the NS group. NS, normal saline; ACBP, anticancer bioactive peptide; DTX, docetaxel; MIX, ACBP combined with DTX; L-MIX, ACBP combined with low dose DTX.

and L-MIX groups were not significantly different compared with the NS group (Fig. 3B). Hematoxylin \& eosin staining of the liver and spleen revealed no marked cell necrosis, edema or inflammatory cell infiltration in all groups (Fig. 3C and D).

p21, p53 and Ki67 immunohistochemical scores in tumor tissues from nude mice. Immunohistochemical staining displayed lower Ki67 protein expression in the ACBP, DTX, MIX and L-MIX groups compared with the NS group (Fig. 4A). p21 immunohistochemical scores were significantly lower in the MIX and L-MIX groups compared with the NS group $(\mathrm{P}<0.01)$ (Fig. 4B). p21 immunohistochemical scores were also decreased in the ACBP and DTX groups, but the decrease was not significantly different compared with the NS group (Fig. 4B). The p21 immunohistochemical score was significantly reduced in the MIX and L-MIX groups compared with the NS and ACBP groups, but no significant difference was identified between the MIX and L-MIX groups (Fig. 4B).

The p53 immunohistochemical score was significantly decreased in the L-MIX, DTX and MIX groups compared 


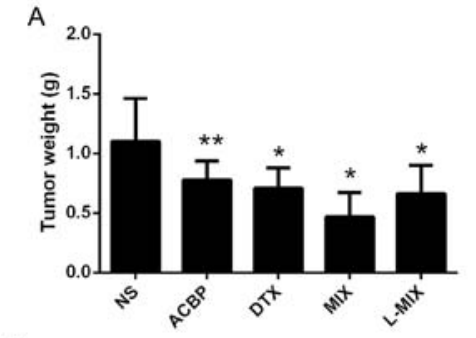

C

NS


DTX $9000 \%$
B

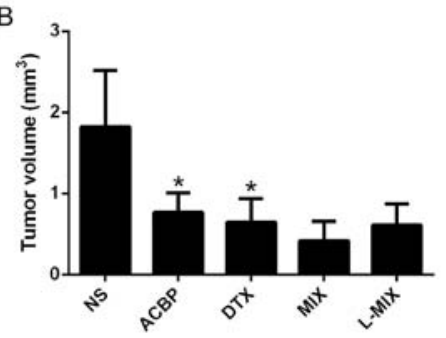

$\operatorname{Mix} \theta 003000$

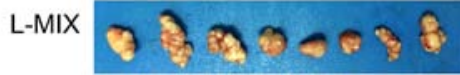

$-10 \mathrm{~mm}$

Figure 2. Measurements of tumor size in tumor-bearing mice. Tumor (A) weight, (B) volume and (C) morphology in tumor-bearing nude mice are presented. ${ }^{*} \mathrm{P}<0.05$ and ${ }^{* *} \mathrm{P}<0.01$ vs. the NS group. NS, normal saline; ACBP, anticancer bioactive peptide; DTX, docetaxel; MIX, ACBP combined with DTX; L-MIX, ACBP combined with low dose DTX.

A

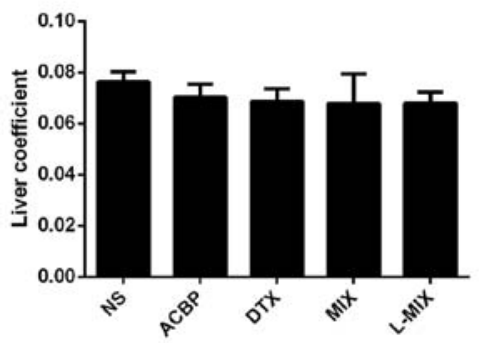

B

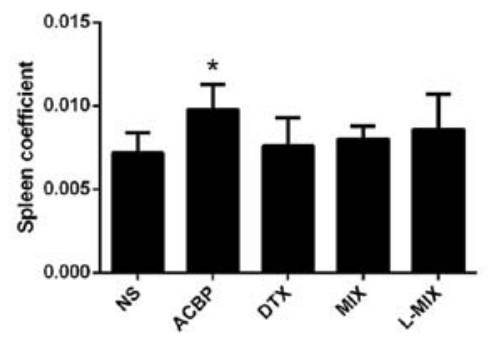

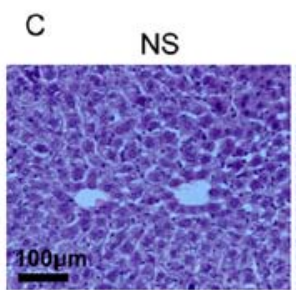

NS

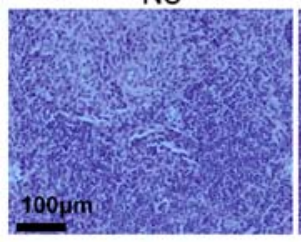

ACBP



ACBP

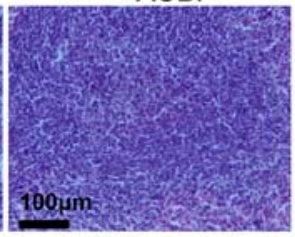



DTX

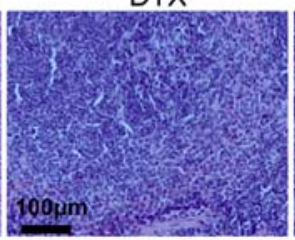

MIX

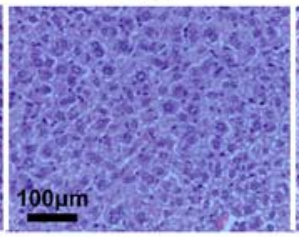

MIX

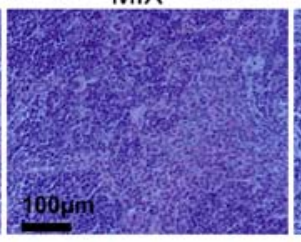

L-MIX

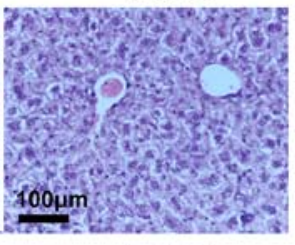

L-MIX

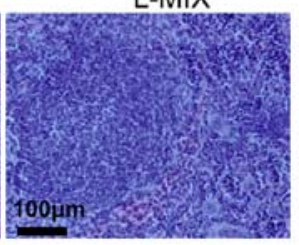

Figure 3. Effect of treatment on the liver and spleen of tumor-bearing mice. The (A) liver coefficient and (B) spleen coefficient. (C) Hematoxylin \& eosin staining of the liver and (D) spleen are presented (magnification, $\mathrm{x} 100$ ). ${ }^{*} \mathrm{P}<0.05 \mathrm{vs}$. the NS group. NS, normal saline; ACBP, anticancer bioactive peptide; DTX, docetaxel; MIX, ACBP combined with DTX; L-MIX, ACBP combined with low dose DTX.

with the NS group $(\mathrm{P}<0.01$; Fig. 4B), but the ACBP group displayed a score that was not significantly different compared with the NS group ( $\mathrm{P}<0.05$; Fig. 4B). The MIX group exhibited a significantly decreased p53 immunohistochemical score compared with the ACBP $(\mathrm{P}<0.01$; Fig. 4B). Furthermore, the L-MIX group displayed a significantly decreased p53 immunohistochemical score compared with the ACBP group ( $\mathrm{P}<0.01$; Fig. 4B). The L-MIX group also exhibited significantly reduced p53 immunohistochemical scores compared with the ACBP, and DTX $(\mathrm{P}<0.05)$.
Ki67 immunohistochemical scores were significantly lower in the four treatment groups compared with the NS group $(\mathrm{P}<0.01$; Fig. 4B). The MIX and L-MIX groups exhibited significantly decreased Ki67 immunohistochemical scores compared with, ACBP and DTX groups $(\mathrm{P}<0.01)$, but there was no significant differences between the MIX and L-MIX groups (Fig. 4B).

Expression of the tumor-associated genes p21, p53 and Ki67. Using GAPDH as an internal reference gene, the relative mRNA levels of p21, p53 and Ki67 were detected by RT-qPCR. 


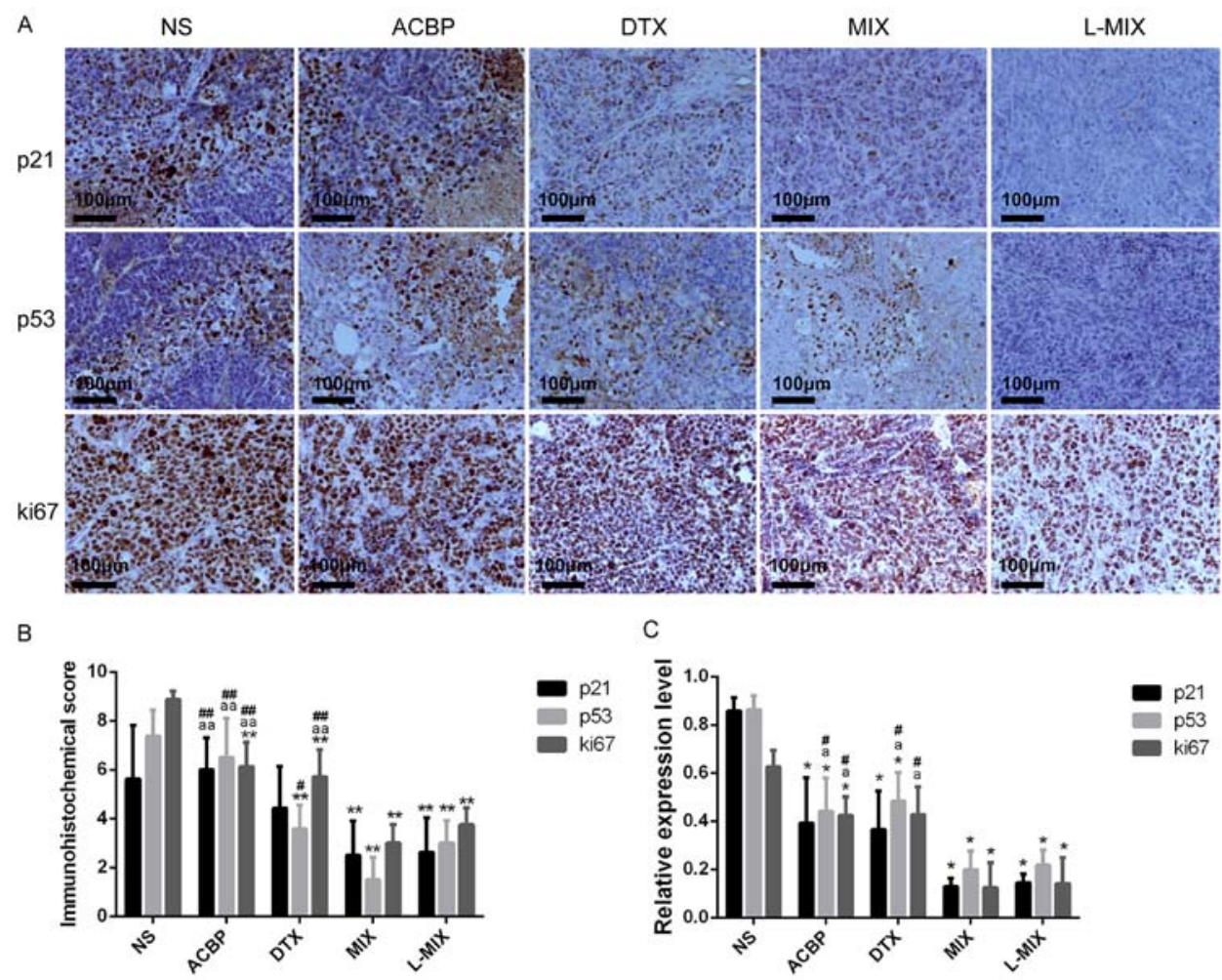

Figure 4. Expression of p21, p53 and Ki67 in tumor tissues. Expression of p21, p53 and Ki67 in tumor tissues by (A) immunohistochemical staining (magnification, $x 100$ ) and (B) immunohistochemical scoring. (C) Relative expression of p21, p53 and Ki67 in tumor tissues, measured by reverse transcription-quantitative PCR. ${ }^{*} \mathrm{P}<0.05$ and ${ }^{* *} \mathrm{P}<0.01$ vs. the NS group. ${ }^{\mathrm{a}} \mathrm{P}<0.05$ and ${ }^{\text {aa }} \mathrm{P}<0.01$ vs. the MIX group. ${ }^{\text {"P }} \mathrm{P}<0.05$ and ${ }^{\# \#} \mathrm{P}<0.01$ vs. the L-MIX group. NS, normal saline; ACBP, anticancer bioactive peptide; DTX, docetaxel; MIX, ACBP combined with DTX; L-MIX, ACBP combined with low dose DTX.

The relative mRNA expression of p21 and p53 was significantly lower in the four treatment groups compared with the NS group $(\mathrm{P}<0.05)$. Regarding $\mathrm{p} 21$, the four treatment groups displayed decreased expression levels compared with the NS group $(\mathrm{P}<0.05)$, but there was no significant difference between the ACBP and DTX groups and the MIX, L-MIX groups. The expression of $\mathrm{p} 53$ was significantly reduced in the four treatment groups compared with the NS group ( $\mathrm{P}<0.05$; Fig. 4C). Furthermore, p53 expression levels were significantly reduced in the MIX and L-MIX groups compared with the ACBP and DTX groups $(\mathrm{P}<0.05$; Fig. $4 \mathrm{C})$, but there was no significant difference between the MIX and L-MIX groups. The ACBP, MIX and L-MIX groups exhibited significantly reduced Ki67 gene expression compared with the NS group ( $\mathrm{P}<0.05$; Fig. 4C). However, there was no significant difference in the expression of Ki67 between the DTX and NS groups (Fig. 4C). The MIX and L-MIX groups displayed significantly decreased Ki67 levels compared with the ACBP and DTX groups $(\mathrm{P}<0.05$; Fig. 4C). However, there was no significant difference in Ki67 expression between the MIX and L-MIX groups (Fig. 4C).

\section{Discussion}

Neoadjuvant and adjuvant chemotherapy have become routine treatment strategies for breast cancer. Approximately $81.4 \%$ of patients with invasive breast cancer are treated with chemotherapy (29). At present, chemotherapy is not effective and is often limited as the majority of tumors have pre-existing resistance mediators (30). Furthermore, treatment prior to chemotherapy is often ineffective and initial chemotherapeutic drugs decrease in effectiveness over the course of the treatment, eventually leading to disease progression and tumor recurrence (30). Common complications of chemotherapeutic drugs are nausea, vomiting, phlebitis, alopecia, oral mucositis and bone marrow suppression (31). A large number of studies have reported ovarian failure (mainly menstrual atresia) and cognitive impairment caused by breast cancer chemotherapy, resulting in physical and mental complications in young and middle-aged female patients (31-33). Therefore, the present study aimed to identify a novel drug that enhanced the sensitivity and reduced the associated toxic and adverse effects of chemotherapeutic drugs.

In addition to the more commonly used methods of clinical surgery, radiotherapy, chemotherapy and endocrine therapy, the use of biotherapy as a cancer treatment is increasing (34). It has been reported that ACBP treatment can effectively inhibit the human breast cancer cell line MDA-MB-231, inhibit tumor cell proliferation, induce apoptosis and block cell cycle progression (35). Furthermore, ACBP can activate the immune system to improve the immune function of the human body and achieve an antitumor effect (35). In addition, numerous studies have suggested that ACBP regulates energy consumption and alleviates digestive tract toxicity $(18-20,22)$. Therefore, ACBP treatment may be potentially decrease the toxic side effects of DTX.

According to the hematoxylin \& eosin staining in the present study, the hepatocytes in each treatment group were neatly arranged and even in size and there was no obvious inflammatory cell infiltration, fibrosis or necrosis in the hepatocytes. However this may be due to the short period of intermittent drug administration. Additionally, there were no significant differences in 
the liver coefficients between the groups of tumor-bearing nude mice, which also indicated that the short-term intermittent use of ACBP did not have a toxic effect on the liver. The spleen is the largest immune organ and is primarily involved in humoral immunity (36). The spleen coefficient can reflect the immune status of the individual; the higher the spleen coefficient is, the stronger the immunity of the individual in the absence of other diseases (36). The spleen coefficient was significantly higher in the ACBP group compared with control groups $(\mathrm{P}<0.05)$. The results indicated that ACBP may enhance the immunity of tumor-bearing nude mice.

Breast cancer is a highly heterogeneous disease, which can be divided into four types according to the expression of the estrogen receptor, progesterone receptor, human epidermal growth factor receptor-2 (HER2) and Ki-67, detected by immunohistochemistry. The four types are luminal A, luminal B, HER2-positive and triple-negative breast cancer (TNBC) (37). These classifications provide reliable guidance for the individualized treatment of breast cancer and the prediction of prognosis at the molecular level (37). The p53 gene is the most widely studied tumor suppressor gene (38). The p21 gene is a product of the Ras proto-oncogene and its upregulation is closely associated with the development of colorectal cancer (39). However, the relationship between p21 and breast cancer remains unclear. Ki67 is recognized as a breast cancer proliferation factor as Ki67 expression and breast cancer cell proliferation, malignancy degree, invasiveness and distant metastasis are positively correlated (40). The MDM2-p53-p21 signaling pathway is one of the most important signaling pathways involving the p53 gene. Alterations in the function or structure of any gene in this signaling pathway can result in tumor formation (41). p21 is located downstream of p53 and depends primarily on p53 activity during cell senescence, cell cycle regulation and apoptosis (41). The human breast cancer cell line MDA-MB-231 used in the present study is a TNBC with a high tumor-formation rate, high degree of malignancy, strong invasiveness and poor prognosis (42).

In the present study, p53 and p 21 levels were not consistent at the level of protein and mRNA expression. The mRNA expression of p53 and p21 was significantly lower in tumor-bearing nude mice treated with ACBP and DTX compared with NS treated mice. From the results, it may be speculated that the anticancer effect of ACBP may occur via the regulation of p53 and p21 gene expression. Therefore, ACBP may increase the rate of apoptosis induced by tumor cells and regulate cell cycle progression in breast cancer cells. High expressions of p53, p21 and Ki67 has been associated with poor prognosis in a number of studies $(40,43,44)$. The expression of the three genes in nude mice treated with ACBP was significantly decreased compared with the NS group. While p53 and Ki67 was significantly lower in the MIX group compared with the ACBP and DTX groups. As a result, ACBP alone had an antitumor effect, but when combined with DTX, ACBP enhanced DTX sensitivity. Regarding quality of life parameters, although the activity levels and weights of the nude mice were not significantly different between the groups, the water consumption in the ACBP group was decreased compared with the NS group $(\mathrm{P}<0.01)$. The tumor weights of the MIX and L-MIX groups were significantly lower compared with the NS groups $(\mathrm{P}<0.05)$, which also suggested that ACBP had an antitumor effect and enhanced DTX sensitivity. The present study identified a novel combined therapy for breast cancer.

To conclude, ACBP effectively inhibited the growth of the human breast cancer cell line MDA-MB-231 in tumor-bearing mice, which not only improved the quality of life of animals but also increased DTX sensitivity. Short-term use of ACBP did not elicit toxic and adverse effects associated with hepatocyte injury, and the increase in the spleen coefficient suggested that the antitumor effect of ACBP might be associated with improvements in immunity of nude mice. The expression of p53, p21 and Ki67 further suggested an antitumor effect for ACBP and indicated that ACBP combined with chemotherapy to reduce DTX toxicity. Therefore, the p53-p21 signaling pathway might serve a key role in the treatment of human breast cancer. The present study suggested a novel approach for the clinical treatment of breast cancer. Further investigation into the mechanisms of action of the antitumor and chemotherapy sensitization effects of ACBP are required.

\section{Acknowledgements}

Not applicable.

\section{Funding}

The present study was supported by the National Natural Science Foundation of China (grant no. 81660468), the Natural Science Foundation of Inner Mongolia Autonomous region (grant no. 2017BS0812) and the General Project of Clinical Medical Research Center, Affiliated Hospital of Inner Mongolia Medical University (grant no. NYFY YB044).

\section{Availability of data and materials}

The datasets used and/or analyzed during the current study are available from the corresponding author on reasonable request.

\section{Authors' contributions}

XS conceived and designed the present study. BG and XL acquired, analyzed and interpreted the data. XL drafted the manuscript. XS agrees to be accountable for the work in ensuring that questions related to the integrity of any part of the work are appropriately investigated and resolved. All authors read and approved the final manuscript.

\section{Ethics approval and consent to participate}

All animal experiments were approved by the Ethics Committee for Animal Experiments of Inner Mongolia Medical College (approval no. YKD2016152).

\section{Patient consent for publication}

Not applicable.

\section{Competing interests}

The authors declare that they have no competing interests. 


\section{References}

1. Song CG, Hu Z, Wu J, Luo JM, Shen ZZ, Huang W and Shao ZM: The prevalence of BRCA1 and BRCA2 mutations in eastern Chinese women with breast cancer. J Cancer Res Clin Oncol 132: 617-626, 2006.

2. Schild SE and Vokes EE: Pathways to improving combined modality therapy for stage III nonsmall-cell lung cancer. Ann Oncol 27: 590-599, 2016.

3. Ye H, Xu HL, Shen Q, Zheng Q and Chen P: Palliative resection of primary tumor in metastatic nonfunctioning pancreatic neuroendocrine tumors. J Surg Res 243: 578-587, 2019.

4. Ghafoor A, Jemal A, Ward E, Cokkinides V, Smith R and Thun M: Trends in breast cancer by race and ethnicity. Ca Cancer J Clin 53: 342-355, 2003.

5. Feng RM, Zong YN, Cao SM and Xu RH: Current cancer situation in China: Good or bad news from the 2018 global cancer statistics? Cancer Commun (Lond) 39: 22, 2019.

6. Culakova E, Thota R, Poniewierski MS, Kuderer NM, Wogu AF, Dale DC, Crawford J and Lyman GH: Patterns of chemotherapy-associated toxicity and supportive care in US oncology practice: A nationwide prospective cohort study. Cancer Med 3: 434-444, 2014

7. Verbelen H, Tjalma W, Meirte $\mathbf{J}$ and Gebruers N: Long-term morbidity after a negative sentinel node in breast cancer patients. Eur J Cancer Care (Engl) 28: e13077, 2019.

8. Le DL, Cao H and Yang LX: Cardiotoxicity of molecular-targeted drug therapy. Anticancer Res 34: 3243-3249, 2014.

9. Feng QJ, Zhang F, Huang XY and Wu ZX: Effectiveness and complications of anthracycline and taxane in the therapy of breast cancer: A meta-analysis. Pathol Oncol Res 20: 179-184, 2014.

10. Rivera E and Cianfrocca M: Overview of neuropathy associated with taxanes for the treatment of metastatic breast cancer. Cancer Chemother Pharmacol 75: 659-670, 2015.

11. Andreopoulou E and Sparano JA: Chemotherapy in patients with anthracycline- and taxane-pretreated metastatic breast cancer: An overview. Curr Breast Cancer Rep 5: 42-50, 2013.

12. Kayl AE and Meyers CA: Side-effects of chemotherapy and quality of life in ovarian and breast cancer patients. Curr Opin Obstet Gynecol 18: 24-28, 2006.

13. Yavari B, Mahjub R, Saidijam M, Raigani M and Soleimani M: The potential use of peptides in cancer treatment. Curr Protein Pept Sci 19: 759-770, 2018.

14. Albenzio M, Santillo A, Caroprese M, Della Malva A and Marino R: Bioactive peptides in animal food products. Foods 6: E35, 2017.

15. Bhat ZF, Kumar S and Bhat HF: Antihypertensive peptides of animal origin: A review. Crit Rev Food Sci Nutr 57: 566-578, 2017.

16. Cicero AFG, Fogacci F and Colletti A: Potential role of bioactive peptides in prevention and treatment of chronic diseases: A narrative review. Br J Pharmacol 174: 1378-1394, 2017.

17. Hilchie AL, Hoskin DW and Power Coombs MR: Anticancer activities of natural and synthetic peptides. Adv Exp Med Biol 1117: 131-147, 2019.

18. Xing Z, Yu L, Li X and Su X: Anticancer bioactive peptide-3 inhibits human gastric cancer growth by targeting miR-338-5p Cell Biosci 6: 53, 2016.

19. Su X, Dong C, Zhang J, Su L, Wang X, Cui H and Chen Z: Combination therapy of anti-cancer bioactive peptide with cisplatin decreases chemotherapy dosing and toxicity to improve the quality of life in xenograft nude mice bearing human gastric cancer. Cell Biosci 4: 7, 2014

20. Li X, Xia L, Ouyang X, Suyila Q, Su L and Su X: Bioactive peptides sensitize cells to anticancer effects of oxaliplatin in human colorectal cancer xenografts in nude mice. Protein Pept Lett 26: 512-522, 2019.

21. Ma J, Hu X, Li J, Wu D, Lan Q, Wang Q, Tian S and Dong W: Enhancing conventional chemotherapy drug cisplatin-induced anti-tumor effects on human gastric cancer cells both in vitro and in vivo by thymoquinone targeting PTEN gene. Oncotarget 8 : 85926-85939, 2017.

22. Yu L, Yang L, An W and Su X: Anticancer bioactive peptide-3 inhibits human gastric cancer growth by suppressing gastric cancer stem cells. J Cell Biochem 115: 697-711, 2014.

23. Zhang S, Guan J, Sun M, Zhang D, Zhang H, Sun B, Guo W, Lin B, Wang Y, He Z, et al: Self-delivering prodrug-nanoassemblies fabricated by disulfide bond bridged oleate prodrug of docetaxel for breast cancer therapy. Drug Deliv 24: 1460-1469, 2017.
24. Chi CL,Li FW, Liu HB, Feng SY, Zhang YJ, Zhou D and Zhang RG: Docetaxel-loaded biomimetic nanoparticles for targeted lung cancer therapy in vivo. J Nanoparticle Res 21: 144, 2019.

25. Li C, Wang Z, Wang Q, Ka Yan Ho RL, Huang Y, Chow MSS Kei Lam CW and Zuo Z: Enhanced anti-tumor efficacy and mechanisms associated with docetaxel-piperine combinationin vitro and in vivo investigation using a taxane-resistant prostate cancer model. Oncotarget 9: 3338-3352, 2017.

26. Grover A, Hirani A, Pathak Y and Sutariya V: Brain-targeted delivery of docetaxel by glutathione-coated nanoparticles for brain cancer. AAPS PharmSciTech 15: 1562-1568, 2014.

27. Stacul F, Bertolotto M, De Gobbis F, Calderan L, Cioffi V, Romano A, Zanconati F and Cova MA: US colour-Doppler US and fine-needle aspiration biopsy in the diagnosis of thyroid nodules. Radiol Med 112: 751-762, 2007 (In English, Italian).

28. Livak KJ and Schmittgen TD: Analysis of relative gene expression data using real-time quantitative PCR and the 2(-Delta Delta C(T)) method. Methods 25: 402-408, 2001

29. Fan L, Strasser-Weippl K, Li JJ, St Louis J, Finkelstein DM, Yu KD, Chen WQ, Shao ZM and Goss PE: Breast cancer in China. Lancet Oncol 15: e279-e289, 2014.

30. Demichele A, Yee D and Esserman L: Mechanisms of resistance to neoadjuvant chemotherapy in breast cancer. N Engl J Med 377: 2287-2289, 2017.

31. Morarji K, Mcardle O, Hui K, Gingras-Hill G, Ahmed S, Greenblatt EM, Warner E, Sridhar S, Ali AMF, Azad A and Hodgson DC: Ovarian function after chemotherapy in young breast cancer survivors. Curr Oncol 24: e494-e502, 2017.

32. Witlox L, Schagen SB, de Ruiter MB, Geerlings MI, Peeters PHM, Koevoets EW, van der Wall E, Stuiver M, Sonke G, Velthuis MJ, et al: Effect of physical exercise on cognitive function and brain measures after chemotherapy in patients with breast cancer (PAM study): Protocol of a randomised controlled trial. BMJ Open 9: e028117, 2019.

33. Gokal K: Effects of physical activity on cognitive and psychosocial functioning in breast cancer patients undergoing chemotherapy: A randomised controlled trial (unpublished $\mathrm{PhD}$ thesis). Loughborough University, 2015.

34. Gan X and Wang L: Current situation and prospect of biotherapy of metastatic renal cell carcinoma. Chin J Cancer Biother, 2018 (In Chinese).

35. Jia Q, Wang W and Su X: Inhibitory effect of anticancer bioactive peptides on proliferation of human breast cancer nm 231 cell. J 270-275, 2007.

36. Xu M, Lu JG and Ma QJ: Research on splenic nerve and spleen Immune function. Prog Mod Biomed 10: 2177-2179, 2010.

37. Cheang MC, Chia SK, Voduc D, Gao D, Leung S, Snider J, Watson M, Davies S, Bernard PS, Parker JS, et al: Ki67 index, HER2 status, and prognosis of patients with luminal B breast cancer. J Natl Cancer Inst 101: 736-750, 2009.

38. Li Q, Zhu Y, Hou L, Wang J, Hu G, Fang X, Hu Y, Tao T, Wei X, Tang H, et al: C23 promotes tumorigenesis via suppressing p53 activity. Oncotarget 7: 58274-58285, 2014.

39. Bai S, Feng Q, Pan XY, Zou H, Chen HB, Wang P, Zhou XL, Hong YL, Song SL and Yang JL: Overexpression of wild-type p21Ras plays a prominent role in colorectal cancer. Int J Mol Med 39: 861-868, 2017.

40. Koopman T, Buikema HJ, Hollema H, de Bock GH and van der Vegt B: Digital image analysis of Ki67 proliferation index in breast cancer using virtual dual staining on whole tissue sections: Clinical validation and inter-platform agreement. Breast Cancer Res Treat 169: 33-42, 2018.

41. Morrison CD, Allington TM, Thompson CL, Gilmore HL, Chang JC, Keri RA and Schiemann WP: c-Abl inhibits breast cancer tumorigenesis through reactivation of p53-mediated p21 expression. Oncotarget 7: 72777-72794, 2016.

42. Wang K, Xie SM, He JJ, Ren Y, Xia HB and Zhang XW: Establishment of a bioluminescent MDA-MB-231 cell line for in vivo imaging of human triple-negative breast cancer xenograft. Nan Fang Yi Ke Da Xue Xue Bao 31: 1812-1818, 2011 (In Chinese).

43. Dumay A, Feugeas JP, Wittmer E, Lehmann-Che J, Bertheau P, Espié M, Plassa LF, Cottu P, Marty M, André F, et al: Distinct tumor protein p53 mutants in breast cancers subgroups. Int J Cancer 132: 1227-1231, 2013.

44. Curtis C, Shah SP, Chin SF, Turashvili G, Rueda OM, Dunning MJ, Speed D, Lynch AG, Samarajiwa S, Yuan Y, et al: The genomic and transcriptomic architecture of 2,000 breast tumours reveals novel subgroups. Nature 486: 346-352, 2012.

This work is licensed under a Creative Commons Attribution-NonCommercial-NoDerivatives 4.0 International (CC BY-NC-ND 4.0) License. 